\title{
RICE CROP MAPPING USING SENTINEL-1 TIME SERIES IMAGES (CASE STUDY: MAZANDARAN, IRAN)
}

\author{
Mohammad Saadat ${ }^{1}$, Mahdi Hasanlou ${ }^{1}$ and Saeid Homayouni ${ }^{2}$ \\ ${ }^{1}$ School of Surveying and Geospatial Engineering, College of Engineering, University of Tehran, Iran \\ ${ }^{2}$ Centre Eau Terre Environnement, INRS-Quebec, Canada \\ mohammad.saadat@ut.ac.ir,hasanlou@ut.ac.ir and saeid.homayouni@inrs.ca
}

KEYWORDS: Rice; Sentinel-1; Random forest; Iran; Time series images; Classification

\begin{abstract}
:
Policymaking and planning agricultural improvement require accurate and timely information and statistics. In Iran, collecting and acquiring agricultural statistics is often done in the traditional methods. Related studies have proved that these methods mostly contain some mistakes. Multi-temporal acquisition strategies of remotely sensed data provide an opportunity to improve rice monitoring and mapping. Studying and monitoring rice paddies in vast areas is limited by the presence of cloud cover, the spatial and temporal resolution of optical sensors, and the lack of open access or systematic Radar data. Sentinel-1 satellite data, which are free to access and has a high quality of spatial and temporal resolution, can provide a great opportunity for monitoring crop products, especially rice. In this study, Sigma Nought, Gamma Nought and Beta Nought time series of Sentinel-1 data in VV, VH and VV+VH polarizations were employed for extracting areas under rice cultivation in the region of Mazandaran province, Iran. These satellite data are taken regularly every 12 days, according to the season of the region, from March 21st to September 22nd of 2018. In this study, in order to specify the rice paddies area, several fieldworks were randomly carried out for two weeks, and field data were collected as well. Field data including rice paddies areas and non-rice areas were collected as 'Test and Train data set' and then the Random Forrest (RF) algorithm was carried out to determine the rice paddies area. The classification result was validated using test samples. The accuracy of all classifications results are over $80 \%$ and the best result is related to Sigma Nought and gamma Nought of VH polarization, with an accuracy of $91.37 \%$. The results showed a high capability to evaluate and monitor rice production at moderate levels in a vast area which is regularly exposed to the cloud cover.
\end{abstract}

\section{INTRODUCTION}

Asia is one of the largest producers of rice, and the rice is cultivated in these lands for thousands of years. Rice is also one of the main agricultural product and the main source of food for about half of the world's population (FAOSTAT Database., 2018). In many countries, rice is the foundation of the economy and provides the main food for the people. Nowadays, the rapid population growth increasingly pressures food resources. To control and maintain the balance between rice production and food demands, an effective program for monitoring rice at the regional, national and international levels is required.

Rice is one of the most important foods in Iran in recent years. The extent and distribution of rice paddies in the northern part of Iran compared with other regions is higher, so that more than $70 \%$ of the rice cultivation area (paddies) in Iran is located in the coastal areas of the Caspian Sea and in the provinces of Gilan and Mazandaran. In general, rice production can be implemented in two ways: (1) Irrigated paddies of rice, in which rice paddies are flooded through dams and rivers, and rice can be cultivated more than once a year; (2). Rainfed paddies of rice, in which paddies are irrigated with rainfall and rice is cultivated once a year, followed by secondary crop cultivation (Toan et al., 1997). In the past few years, two main methods have been used for rice paddies mapping: (1) by using manual coordination of cadastral maps, and (2) by using manual digitization based on the visual interpretation of high resolution optical and Near-Infrared (NIR) satellite images (Nguyen et al., 2016). These methods are time-consuming and expensive. Traditional methods of obtaining information from the distribution of rice systems are based on statistical information. However, statistical data can be easily mistaken by human factors, and it is difficult to identify spatial distribution in administrative units of government. The potential of using remote sensing to extract the rice distribution has been well proved and is now widely used for rice mapping (Toan et al., 1997). Estimation of different levels of agricultural products in Iran is usually carried out in three ways including expertizing methods, the estimation method through the inventory and utilizing of new technologies. The precision of the expertizing methods is very low and its results cannot be used in the important decision-making process. In the estimation method through the inventory, although the cropping area is estimated, the product distribution cannot be estimated. The third method is the employing of new technologies, such as remote 
sensing and GIS. What is clear in Iran is the lack of these kinds of data. This lack of information is not only about rice, but also about most of the agricultural crops in Iran, and the need for further studies in this field is necessary. Water and heat requirement of rice is high. Most rice paddies are located in hot and humid areas around the world. Humid environments are usually covered with clouds, so it is difficult to obtain optical remotely sensed data in rice growing areas. Radar data and especially SAR data are working in all kinds of weather conditions and do not require sunlight (Yan, Hao et al., 1998). These images with the capability to capture and view frequencies are an important source for forecasting and displaying rice in tropical regions. In the case of rice, microwave observations depend on the stages of growth, biomass development, plant height, soil moisture, and the duration and frequency of flooding. During the crop rotation period, the presence of background soil surface associated with rice paddies reduces backscattering. While tillering rate, amount of biomass and rice straw are increased, the rate of backscattering response, as a result of increased interaction, increases as well, and the rate of backscattering when the rice reaches to its maximum growth rate and when it is harvested leads to a decrease in backscattering. This has led SAR images to be particularly useful, in view of the range of dynamics and the scattering mechanism of spectral reflection of rice throughout its life cycle, to map of rice cultivation area, the rate of flooding of rice paddies, and the intensity of cultivation (Toan et al. 1997; Ribbes 1999).

\section{MATERIALS AND METHODS}

\subsection{Study Areas}

In Iran, different vareties of rice are cultivated, which they are specifically cultivated in Iran and has a high demand among Iranian consumers whose main priority is Iranian rice to consume. Iran is a large country in the southwest of Asia (in the Middle East) with an estimated area of 1,648,195 $\mathrm{km}^{2}$, and historical records have shown that since the firstcentury of A.C. rice has been cultivated in Iran, and now Iran is a medium rice producer. Only $3 \%$ of the annual cultivated areas in Iran are rice-cultivated paddies and about $70 \%$ of the rice cultivation area is located in the northern provinces of Gilan and Mazandaran, which are located near the Caspian Sea. Iranian rice is transplanted instantly after the Iranian New Year (Nowruz) in late May and is harvested in late August using hand-held or mechanical methods. The study area is located in the eastern region of Mazandaran province in the northern UTM zone of 39 with an area of $73.23 \mathrm{~km}^{2}$ and in this area rice growth stages, from transplanting to harvesting, last from 85 to 100 days (Fig. 2 (a)).

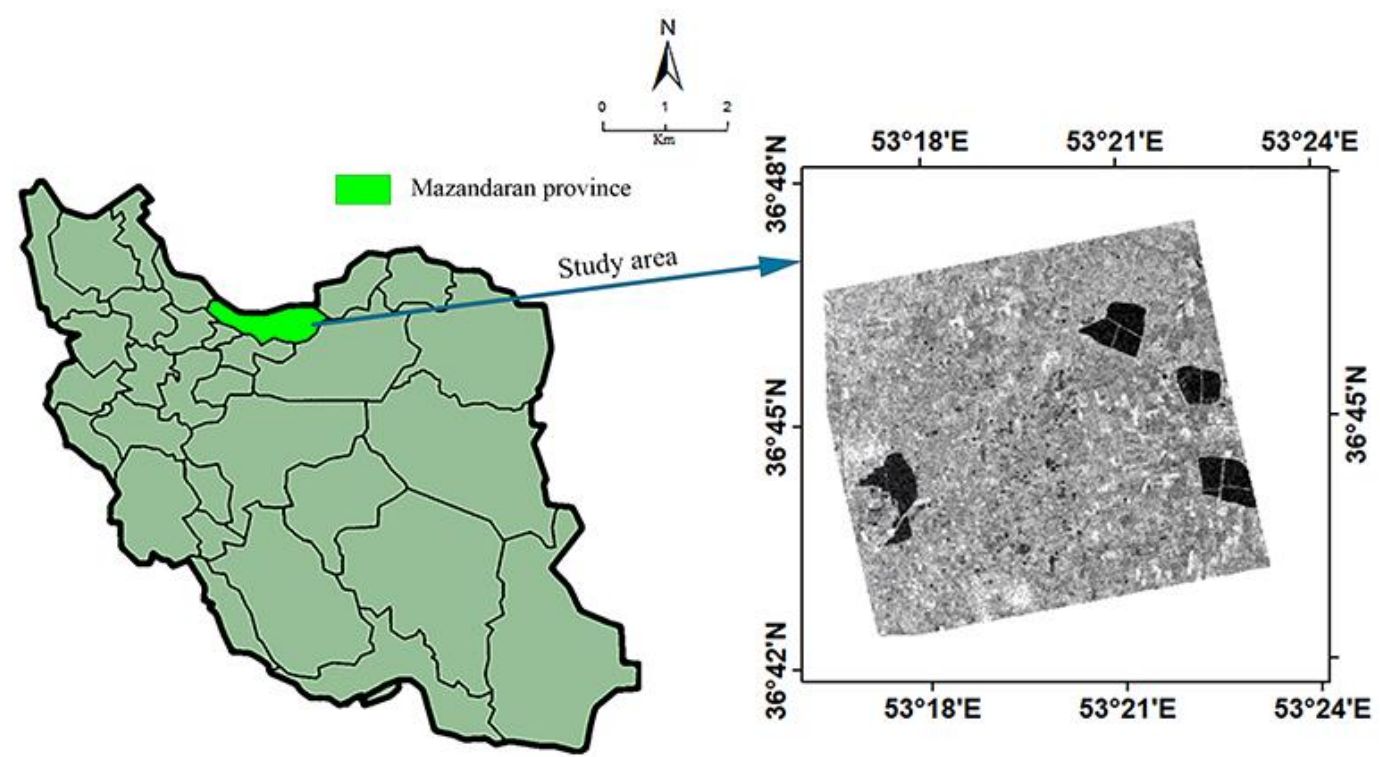

Figure 1. Study area and location of the field survey sites in the Mazandaran province, Iran.

\subsection{Sentinel-1 data and Pre-processing}

Due to the bad weather conditions and the cloudiness of most of the days in the study area, we had to use Sentinel-1 radar images. The Sentinel-1A satellite platform carries a $\mathrm{C}$ band sensor at $5.405 \mathrm{GHz}$ with an incident angle between 20 and 45 degrees. This platform moves in a Sun-synchronous orbit near the pole at an altitude of 693 kilometres. The A1 platform has a 12-day repeat cycle in the equator. The Sentinel-1B platform was launched in April 2016 and is currently collecting data on a regular basis. In this research, the time series of Sentinel-1 images from March 21th to September 22nd of 2018, was acquired every 12 days (including 15 images) (Fig. 2 (b)); the required preprocessing is shown in Fig. 3. 
(a)

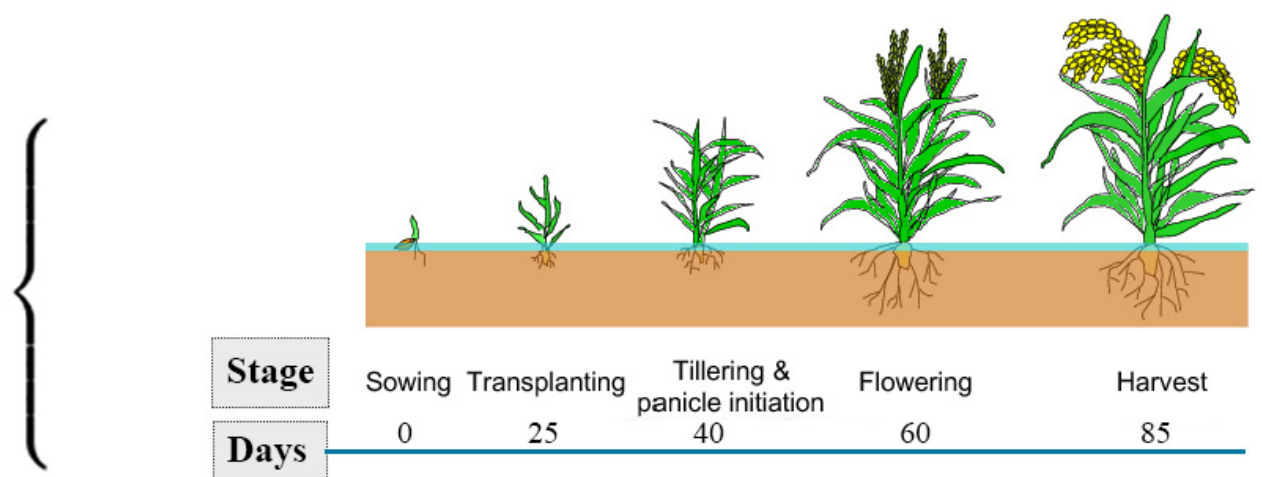

(b)

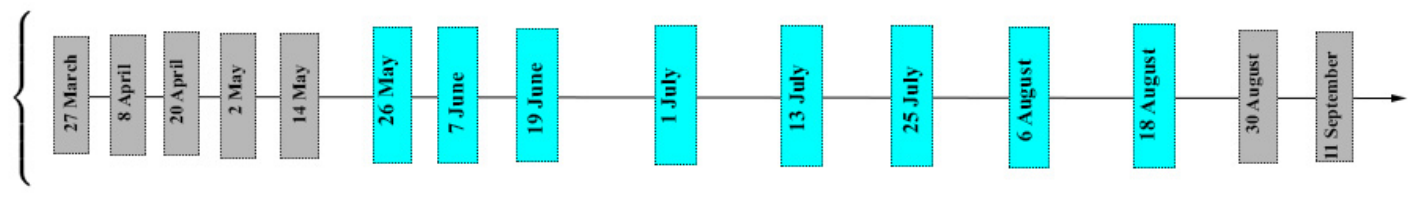

Figure 2. (a) Growth stages of rice, (b) dates for taking images.

To eliminate speckle noise of Sentinel-1 images, the improved Lee filter (Window $3 \times 3$ ) was used. The L1 products (Sentinel-1) are provided with dedicated Calibration Annotation Data Set (CADS) providing the necessary information to convert the radar reflectivity into physical units. The CADS provides four Look Up Tables (LUTs):

$$
A_{\beta}
$$

$\beta$ (Beta Nought): to transform the radar reflectivity into beta $\beta 0$ where the area normalization is aligned with the slant range.

$A_{\sigma}$ (Sigma Nought): to transform the radar reflectivity into radar cross-section $\sigma 0$ where the area normalization is aligned with ground range plane.

$$
A_{\gamma}
$$

$\gamma$ (Gamma Nought): to transform the radar reflectivity into gamma $\gamma 0$ where the area normalization is aligned with a plane perpendicular to slant range.

\begin{abstract}
$A_{d n}$ : to revert for the final pixel scaling. The final products are coded in 16 bits' integers (signed for SLC and unsigned for GRD). Those final products are generated from the same internal SLC product coded in floats. In order to best use the limited integer dynamic range, the internal SLC is scaled. $A_{d n}$ defines the final scaling from internal SLC to the final product (Radiometric Calibration of S-1 Level 1 Products Generated by the S-1 IPF).

Therefore, the DN of Sentinel-1 images were converted into Sigma Nought, Beta Nought, and Gamma Nought. In this study, time series of sigma Nought, beta Nought and gamma Nought images of level 1 images of Sentinel-1 were used using Random Forest (RF) algorithm to determine the areas under rice cultivation (Fig. 3).
\end{abstract}

\subsection{Rice Cropping System Samples}

According to the crop season of the study area, several composites has been set up to produce samples of rice cultivation system. Samples were harvested with the same distribution throughout the study area. A total of 289 samples, including 102 samples of rice cultivating system and 187 non-rice samples were collected (Table 1). These samples were randomly divided into two groups of $30 \%$ and $70 \%$. The first group (70\% of these samples) was used as training samples for determining the rules for monitoring rice harvesting systems. The second group were used to validate the results accuracy.

Table1. Characteristics of training data used for the Classification

\begin{tabular}{ccc}
\hline Class & \# of Polygons & \# of Pixels \\
\hline Rice & 102 & 92,808 \\
Non-Rice & 187 & 109,783 \\
\hline
\end{tabular}

\subsection{Methodology}

The RF classifier consists of a combination of tree classifiers where each classifier is generated using a random vector sampled independently from the input vector, and each tree casts a unit vote for the most popular class to classify an input vector (Breiman 1999). The RF classifier used for this study consists of using randomly selected features or a combination of features at each node to grow a tree. Bagging, a method to generate a training data set by randomly drawing with replacement $N$ examples, where $N$ is the size of the original training set (Breiman 1996), was used for each feature/feature combination selected. Any examples (pixels) are classified by taking the most popular voted class from all the tree predictors in the forest (Breiman 1999). Design of a decision tree required the choice of an attribute selection measure and a pruning method. There are many approaches to the selection of attributes used for decision tree induction and most approaches assign a quality measure directly to the attribute. The most frequently used attribute selection measures in decision tree induction are the 
Information Gain Ratio criterion (Quinlan 1993) and the Gini Index (Breiman et al. 1984). The RF classifier uses the Gini Index as an attribute selection measure, which measures the impurity of an attribute with respect to the classes. For a given training $T$ set, selecting one case (pixel) randomly and expressing that it belongs to class $C_{i}$, so the Gini index can be written as:

$$
\sum \sum_{j \neq i}\left(f\left(C_{i}, T\right) /|T|\right)\left(f\left(C_{j}, T\right) /|T|\right)
$$

where $f(C i, T) / T \mid$ is the probability that the selected case belongs to class $C$. Each time a tree is grown to the maximum depth on new training data using a combination of features. These fully grown trees are not pruned. This is one of the major advantages of the random forest classifier over other decision tree methods like the one proposed by Quinlan (1993). The studies suggest that the choice of the pruning methods, and not the attribute selection measures, affect the performance of tree-based classifiers (Mingers 1989, Pal and Mather 2003). Breiman (1999) suggests that as the number of trees increases, the generalization error always converges even without pruning the tree and overfitting is not a problem because of the Strong Law of Large Numbers (Feller 1968). The number of features used at each node to generate a tree and the number of trees to be grown is two user-defined parameters required to generate a random forest classifier. At each node, only selected features are searched for the best split. Thus, the random forest classifier consists of $N$ trees, where $N$ is the number of trees to be grown, which can be any value defined by the user. To classify a new dataset, each case of the datasets is passed down to each of the $N$ trees. The forest chooses a class having the most out of $N$ votes, for that case.

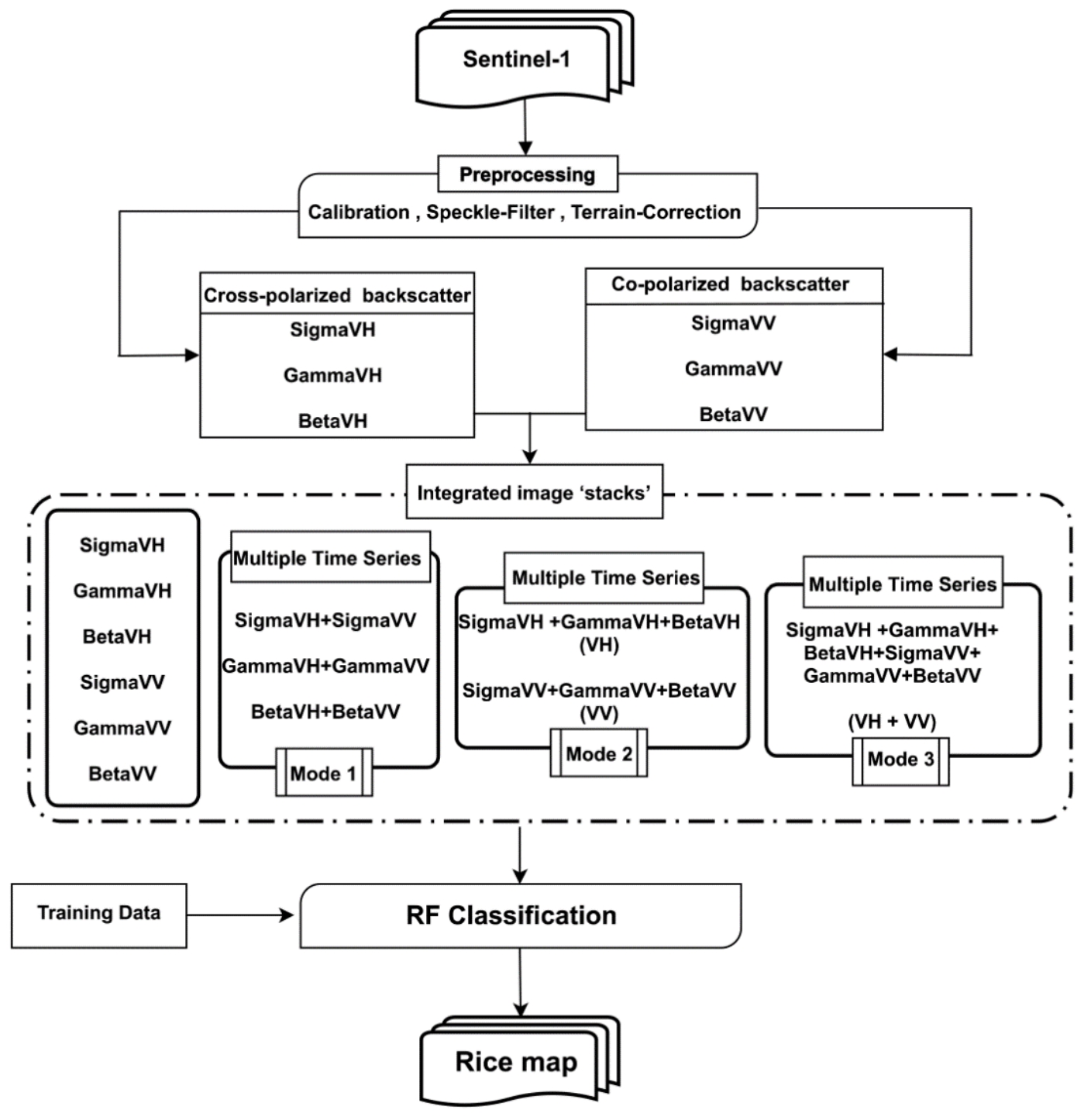

Figure 3. Overview of the methodology for mapping rice areas using the Sentinel-1 time series images. 


\section{RESULTS AND DISCUSSION}

As noted above (Section 2.1), rice is transplanted in the region in late May and harvested in late August. The postdistribution changes of the study area can be obtained by visual interpretation of the images; for example, Figure 4 (a) and Figure $4(b, c)$ are acquired on March $27^{\text {th }}$ and on May $26^{\text {th }}$, respectively (flooding time in the regions for rice cultivation). The flooded areas for rice cultivation can be visually investigated and the changes are extracted, while the post-distribution changes of other areas such as trees, cities, villages, and etc., are negligible and even visually they cannot be clearly observed.

The amount of flooding was mapped using a thresholding technique, in which the ranges of incident angles, seasons and stages of rice growth were also taken into account (Fig. 4 (d)). By integrating flood conditions information with vegetable masks, rice analysis and irrigation can be analyzed more accurately. By analyzing pixel-based time series in a rice growth stages, and by detecting of flood conditions, which was carried out with increased backscattering and minimum dynamic range, rice flooded paddies were identified. This is an effective method for identifying the extent of rice cultivation and the water period (flood duration, flood time and/or flood period), but our purpose in this paper is to identify areas under rice cultivation using ground data. It also should be said that in these types of cultivating patterns, if the Sentinel-1 satellite time series contain some gapes during the key stages, they can potentially cause confusion.
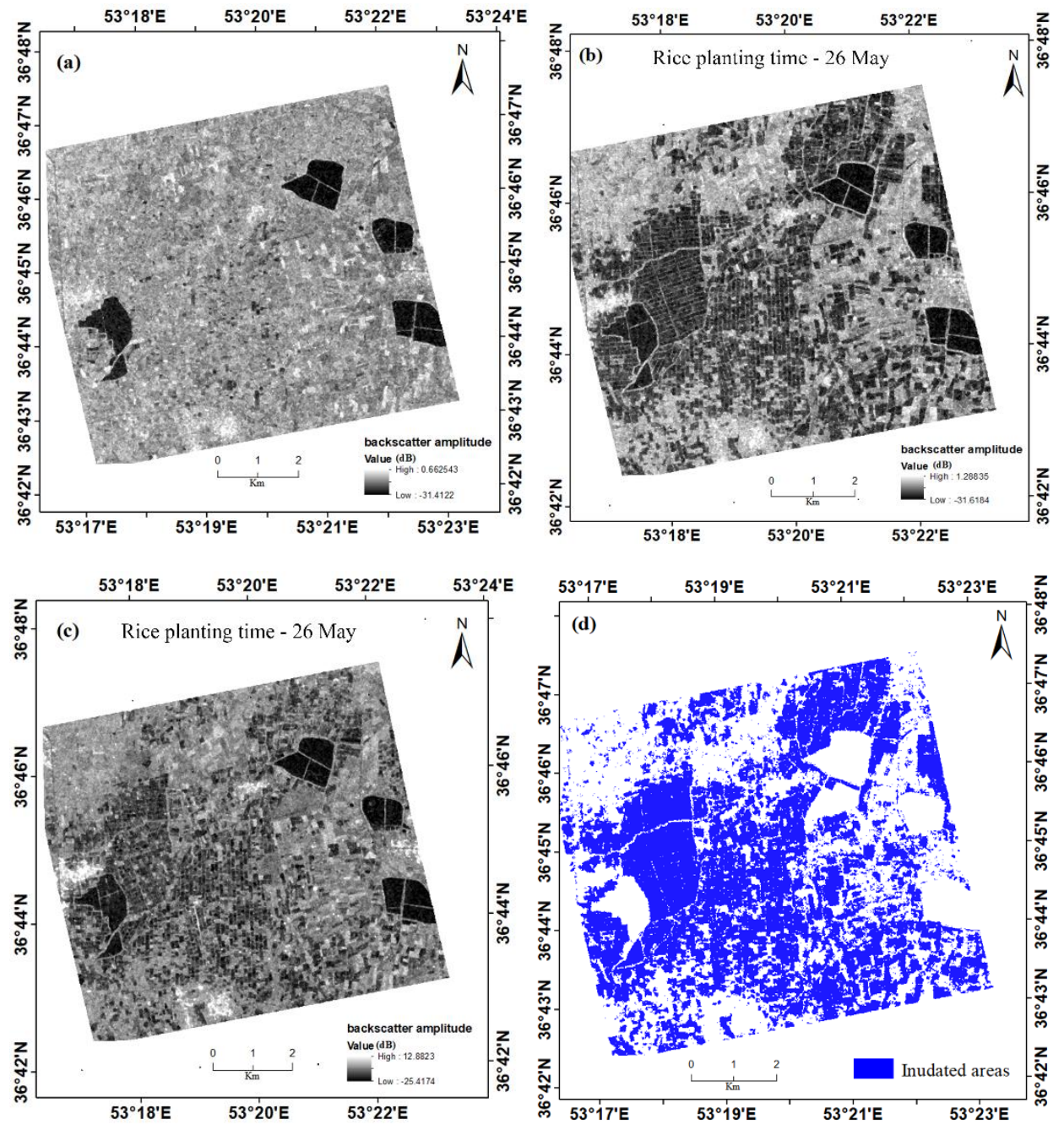

Figure 4. Study area, (a) before planting rice, (b) VH polarization, (c) VV polarization, and (d) inundated areas.

In this paper, the time series of Sigma Nought, Gamma Nought and Beta Nought images derived from the Sentinel1 in polarizations of $\mathrm{VH}, \mathrm{VV}$ and $\mathrm{VV}+\mathrm{VH}$ using the $\mathrm{RF}$ algorithm, have been used to identify rice cultivation areas (Figure 5) and validation for each category was carried out
(Table 2). The results (Fig. 5 and Table 2) showed that VH backscattering is more sensitive to rice growth than $\mathrm{VV}$ backscattering, and this can be clearly seen in Fig. 5 (b, c), and thus, $\mathrm{VH}$ polarization can be much better to identify rice cultivated areas. 

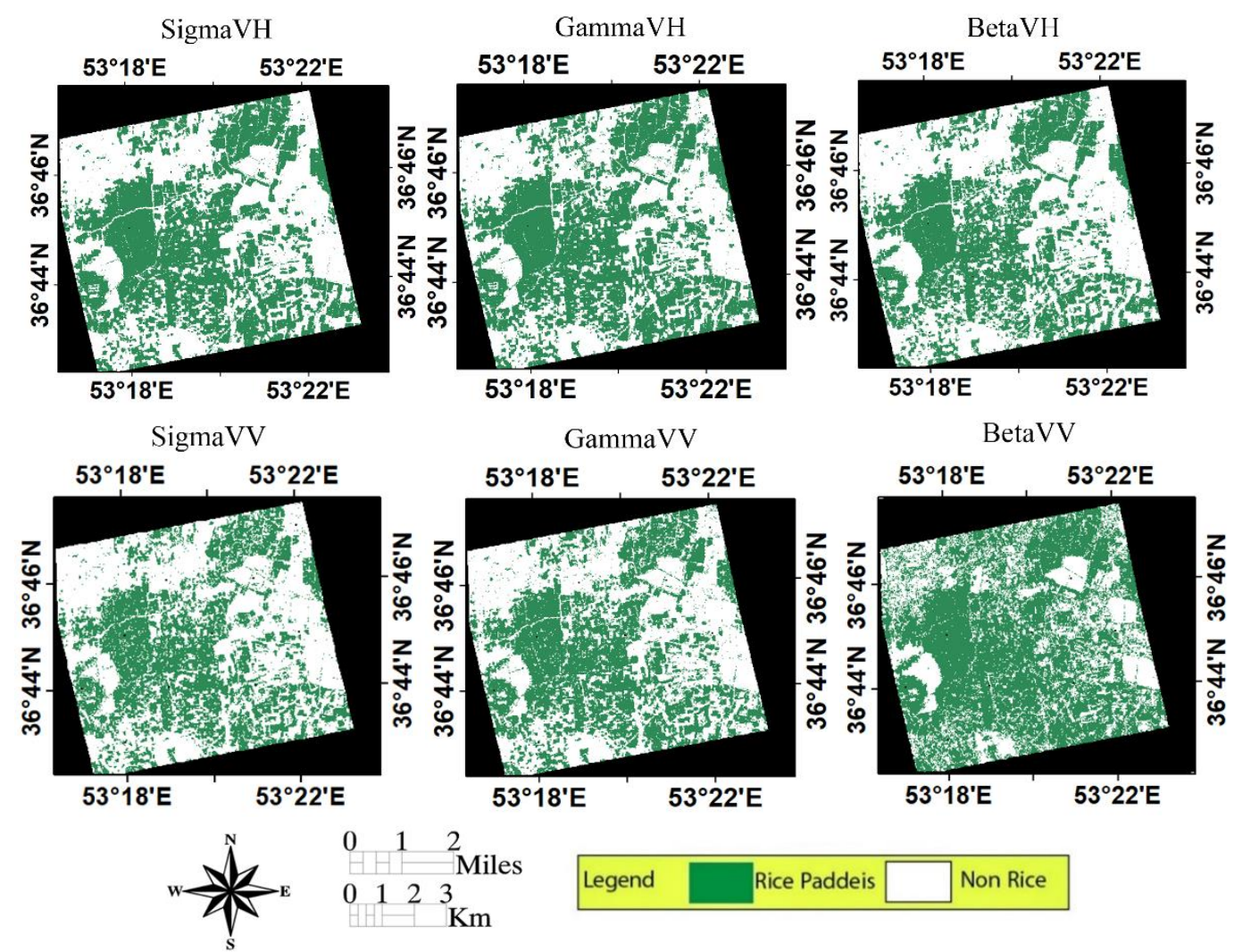

Figure 5. Rice crop map derived from Sentinel-1 time series images.
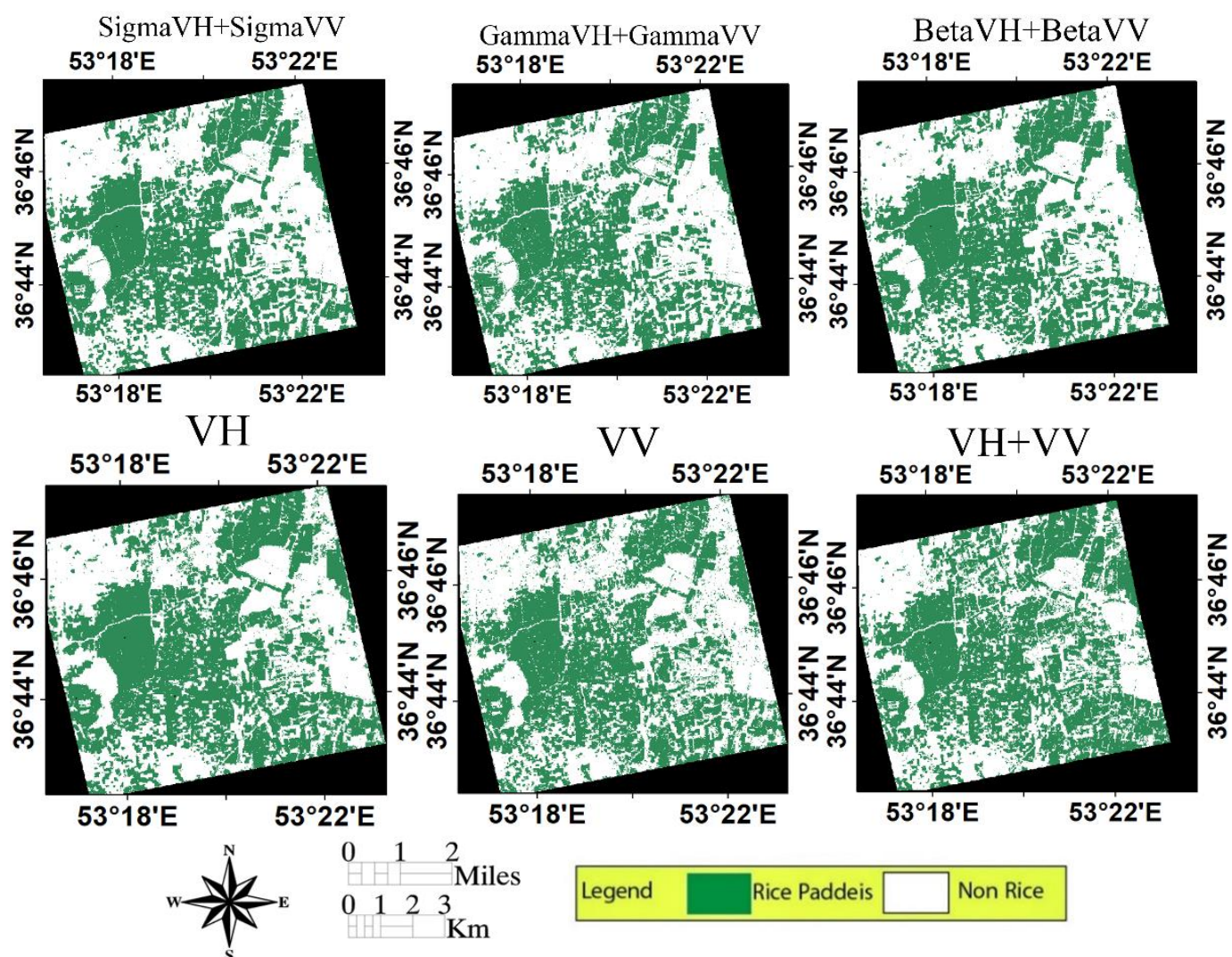

Figure 6. Rice crop map derived from multiple time series of Sentinel-1 images. 
The purpose of this paper is to assess the accuracy of various types of methods using ground data with Sentinel-1 images (Sigma Nought, Gamma Nought and Beta Nought) to obtain the best results for the identification of rice cultivation areas. These methods can be entirely combined and follow a specific principle. Mode (1) shows the combination of $\mathrm{VH}$ and VV polarization sigma Nought, $\mathrm{VH}$ and $\mathrm{VV}$ polarization gamma Nought and VH and VV polarization beta Nought (Fig. 3 (Mode1)). In this case, it can be seen that the accuracies follow an averaging method except for the beta Nought, which their combinations lead to increase their precision rather than to each one of the $\mathrm{VH}$ and $\mathrm{VV}$ polarization beta Nought. Mode (2) is the combination of sigma, gamma, and beta Nought polarizations of VH and VV (Fig. 3 (Mode 2)). In this case, it can also be seen that the $\mathrm{VH}$ polarization uses an averaging method, but in the case of $\mathrm{VV}$ polarization, its precision is the highest precision of the VV polarization (Sigma VV). Mode (3) shows the combination of each one of these images together (Fig. 3 (Mode 3)). In this section, it can be seen that it follows a certain averaging method. finally, the sigma and gamma Nought images of the VH polarization have the highest accuracy. Also, the results of $\mathrm{VH}$ polarization for identifying rice areas cultivation are much more useful than VV polarization (Fig. 6 and Table 2). Changes in the rice areas cultivation can be identified for different situations (Fig. 7).

Table 2. The result of the classification accuracy assessment

\begin{tabular}{cccc}
\hline Number & Data type & OA(\%) & Kapa \\
\hline $\mathbf{1}$ & sigmaVH & 91.37 & 0.82 \\
$\mathbf{2}$ & gammaVH & 91.37 & 0.82 \\
$\mathbf{3}$ & betaVH & 87.93 & 0.75 \\
$\mathbf{4}$ & sigmaVH+ gammaVH +betaVH & 89.65 & 0.79 \\
$\mathbf{5}$ & $(\mathrm{VH})$ & 86.20 & 0.72 \\
$\mathbf{6}$ & sigmaVV & 84.48 & 0.68 \\
$\mathbf{7}$ & gammaVV & 81.03 & 0.62 \\
$\mathbf{8}$ & betaVV & 86.20 & 0.72 \\
$\mathbf{9}$ & sigmaVV+gammaVV+betaVV(VV) & 87.93 & 0.75 \\
$\mathbf{1 0}$ & sigmaVH+sigmaVV & 86.20 & 0.72 \\
$\mathbf{1 1}$ & gammaVH+gammaVV & 89.65 & 0.79 \\
$\mathbf{1 2}$ & betaVH+betaVV & \\
& sigmaVH + gammaVH+betaVH & & 0.79 \\
\hline
\end{tabular}

\begin{tabular}{|r|l|}
\hline 1 & sigmaVH \\
\hline 2 & gamma \\
\hline 3 & betaVH \\
\hline 4 & sigmaVV \\
\hline 5 & gamma \\
\hline 6 & betaVV \\
\hline 7 & sigmaVH+sigmaVV \\
\hline 8 & gammaVH+gammaVV \\
\hline 9 & betaVH+betaVV \\
\hline 10 & VH \\
\hline 11 & VV \\
\hline 12 & VH+VV \\
\hline
\end{tabular}

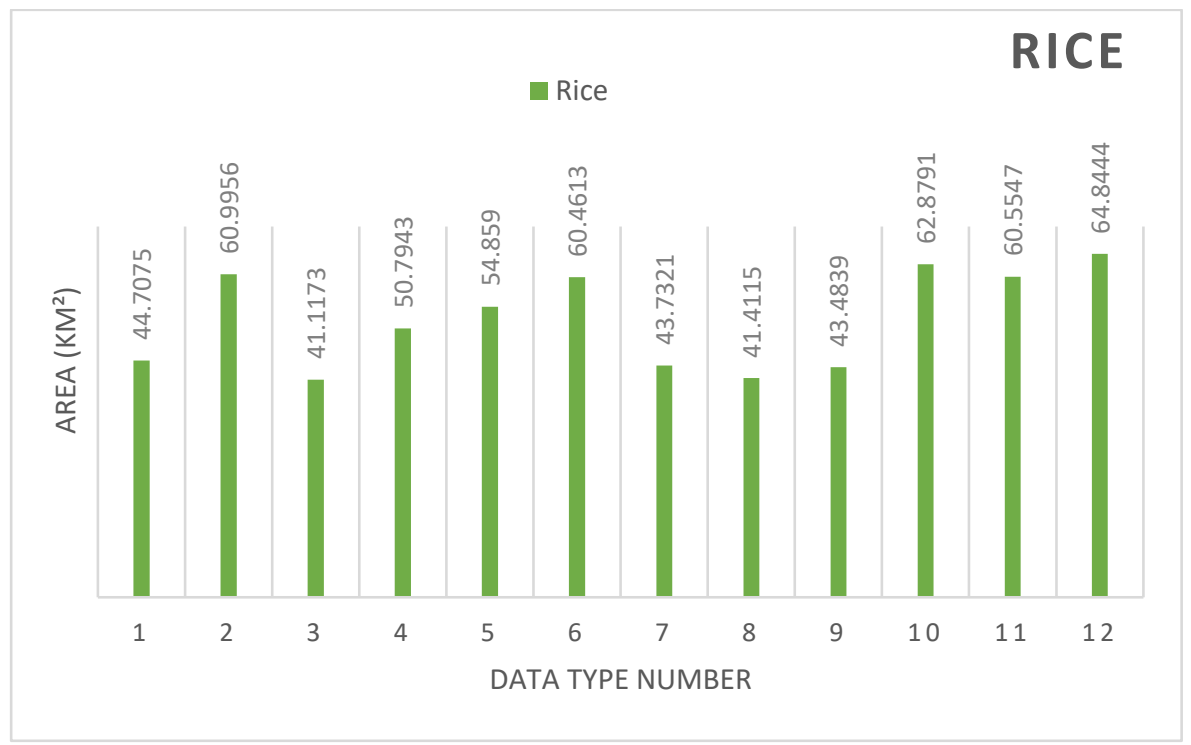

Figure 7. Map of changes in the area of rice paddies.

\section{CONCLUSION}

Various approaches used in the SAR time series allow rice cultivation to be monitored according to the information of the rice paddies extent, flooding conditions, agronomic calendars, and the severity of cropping. Band $\mathrm{C}$ sensitivity of the SAR image to the growth and development of rice crop and the timing of the flooding conditions and its dynamic range is the main stimulus to create a detailed map of rice. It was determined that $\mathrm{VH}$ polarization backscattering is more susceptible to rice growth than VV polarization backscattering, and is much more useful than 
VV polarization for determining areas under rice cultivation. In order to map the rice areas cultivation, several field camps were set up to collect ground data, and then a Random Forrest algorithm with an overall accuracy of $91.37 \%$ and kappa coefficient of $k=0.82$, was carried out in spring and summer 2018. In general, the methodology and results of this study are transparent, robust and scalable and can be implemented in a near-time operational context in Iran, which lack of such information in the agriculture exists.

\section{REFERENCES}

Agricultural Ministry of Iran., 1998. Statistic Book of Agricultural Crops in Mazandaran Province.

FAOSTAT. FAOSTAT Database. Food and Agriculture Organization of the United Nations. 2016. Available online: http://www.fao.org/faostat/en/\#data/QC.

Yan, S., Hao, L. and Huynen, JR., 1998. 7, p.2-10, www.ScienceDirect.com.

Duy Ba Nguyen, Alexander Gruber \& Wolfgang Wagner., 2016. Mapping rice extent and cropping scheme in the Mekong Delta using Sentinel-1A data, Remote Sensing Letters, 7:12, 1209-

1218, DOI: 10.1080/2150704X.2016.1225172.

Toan, T. L., F. Ribbes, L.-F. Wang, N. Floury, K.-H. Ding, J. A. Kong, M. Fujita, and T. Kurosu., 1997. Rice Crop Mapping and Monitoring Using ERS-1 Data Based on Experiment and Modeling Results. IEEE Transactions on Geoscience and Remote Sensing 35 (1): 41 -56. doi: $10.1109 / 36.551933$.

Radiometric Calibration of S-1 Level 1 Products Generated by the S-1 IPF.https://sentinel.esa.int/documents/247904/685163/S1Radiometric-Calibration-V1.0.pdf

Nathan Torbick, William Salas, Diya Chowdhury, Peter Ingraham \& Mai Trinh ., 2017. Mapping rice greenhouse gas emissions in the Red River Delta, Vietnam, Carbon Management, 8:1, 99-

108, DOI: $\underline{10.1080 / 17583004.2016 .1275816}$

Santasombat, Y., 2015. Impact of China's Rise on the Mekong Region. New York: Palgrave Macmillan.

Son, N.-T., C.-F. Chen, C.-R. Chen, H.-N. Duc, and L.-Y, Chang., 2014. A Phenology-Based Classification of Time-Series MODIS Data for Rice Crop Monitoring in Mekong Delta, Vietnam. Remote Sensing 6 (1): 135-156.

Gilbert M, Xiao X M, Chaitaweesub P et al., 2007. Avian influenza, domestic ducks and rice agriculture in Thailand. Agriculture, Ecosystems \& Environment, 119(3/4): 409415.
BREIMAN, L., 1996. Bagging predictors. Machine Learning, 26, pp. 123-140.

BREIMAN, L., 1999. Random forests-random features. Technical Report 567, Statistics Department, University of California, Berkeley, ftp://ftp.stat.berkeley.edu/pub/users/ breiman.

BREIMAN, L., FRIEDMAN, J.H., OLSHEN, R.A. and STONE, C.J., 1984. Classification and Regression Trees (Monterey, CA: Wadsworth).

QUINLAN, J.R., 1993. C4.5: Programs for Machine Learning (San Mateo, CA: Morgan Kaufmann).

FELLER, W., 1968. An Introduction to Probability Theory and its Application, vol.1, 3rd edn (New York: Wiley).

MINGERS, J.Machine Learning., 1989. An empirical comparison of pruning methods for decision tree induction. Machine Learning, 4: 227. https://doi.org/10.1023/A:1022604100933.

PAL, M. and MATHER, P.M., 2003. An assessment of the effectiveness of decision tree methods for land cover classification. Remote Sensing of Environment, 86, pp. 554565 .

PAL, M. and MATHER, P.M., 2003. Support vector classifiers for land cover classification. Map India 2003. New Delhi, 28-31 January, www.gisdevelopment.net/technology/rs/ pdf/23.pdf.

Bazzi, H.; Baghdadi, N.; El Hajj, M.; Zribi, M.; Minh, D.H.T.; Ndikumana, E.; Courault, D.; Belhouchette, H. Mapping Paddy Rice Using Sentinel-1 SAR Time Series in Camargue, France. Remote Sens. 2019, 11, 887. 